\title{
Magnetically-induced outflows from binary neutron star merger remnants
}

\section{Daniel M. Siegel}

Max Planck Institute for Gravitational Physics (Albert Einstein Institute) Am Mühlenberg 1, 14476 Potsdam-Golm, Germany

E-mail: daniel.siegeleaei.mpg.de

\section{Riccardo Ciolfi}

Physics Department, University of Trento

INFN-TIFPA, Trento Institute for Fundamental Physics and Applications

Via Sommarive 14, 38123 Trento, Italy

E-mail: riccardo.ciolfieunitn.it

\begin{abstract}
Recent observations by the Swift satellite have revealed long-lasting $\left(\sim 10^{2}-10^{5} \mathrm{~s}\right)$, "plateaulike" X-ray afterglows in the vast majority of short gamma-ray bursts events. This has put forward the idea of a long-lived millisecond magnetar central engine being generated in a binary neutron star (BNS) merger and being responsible for the sustained energy injection over these timescales ("magnetar model"). We elaborate here on recent simulations that investigate the early evolution of such a merger remnant in general-relativistic magnetohydrodynamics. These simulations reveal very different conditions than those usually assumed for dipole spin-down emission in the magnetar model. In particular, the surrounding of the newly formed NS is polluted by baryons due to a dense, highly magnetized and isotropic wind from the stellar surface that is induced by magnetic field amplification in the interior of the star. The timescales and luminosities of this wind are compatible with early X-ray afterglows, such as the "extended emission". These isotropic winds are a generic feature of BNS merger remnants and thus represent an attractive alternative to current models of early X-ray afterglows. Further implications to BNS mergers and short gamma-ray bursts are discussed.
\end{abstract}

Swift: 10 Years of Discovery,

2-5 December 2014

La Sapienza University, Rome, Italy 


\section{Introduction}

Coalescing binary neutron stars (BNSs) and neutron star-black hole (NS-BH) binaries are not only among the most promising candidates for direct detection of gravitational waves (GWs) with upcoming ground-based interferometers such as advanced LIGO and Virgo [1,2]. The accretion of a remnant torus around a $\mathrm{BH}$ formed promptly after merger also represents the leading scenario to explain the formation of a short gamma-ray burst (SGRB; e.g., $[3,4,5,6])$. However, the recent discovery of long-lasting X-ray afterglows (referred to as "extended emission" and "X-ray plateaus"; e.g., [7, 8]) in the vast majority of SGRB events observed by the Swift satellite [9] indicate ongoing energy injection on timescales of up to $\sim 10^{4} \mathrm{~s}$, which are incompatible with the short accretion timescale of a remnant torus $(\lesssim 1 \mathrm{~s})$.

These X-ray afterglows can, however, be interpreted by assuming that the merger leads to the formation of a long-lived, rapidly rotating NS (a millisecond magnetar; henceforth referred to as the "magnetar model"; e.g., [10, 11, 12, 13]). Despite individual differences in the phenomenology, different variants of the magnetar model have in common that they postulate the formation of a uniformly rotating, highly magnetized NS and that at least the late-time X-ray emission is powered by dipole spin-down radiation, which requires an essentially baryon-free environment around the NS.

The magnetar model challenges the NS-BH progenitor channel for SGRBs, since a magnetar cannot be formed in this case, and we are thus led to consider BNS mergers as the main formation channel. The outcome of a BNS merger depends on the equation of state of nuclear matter at high densities, which is unknown. However, the recent discovery of high-mass neutron stars $[14,15]$ indicate a maximum mass for stable NSs of $M_{\mathrm{TOV}} \gtrsim 2 \mathrm{M}_{\odot}$. Furthermore, the mass distribution of BNSs is peaked around $1.3-1.4 \mathrm{M}_{\odot}$ and this yields an approximate mass of the merger remnant of $\approx 2.4 \mathrm{M}_{\odot}$ [16]. Combining this with the fact that uniform rotation can centrifugally support NSs against gravitational collapse up to masses of $\approx 1.2 M_{\mathrm{TOV}} \gtrsim 2.4 \mathrm{M}_{\odot}$ [17], merger remnants are very likely supramassive. If the merger remnant is only slightly hypermassive (i.e. above the maximum mass for uniformly rotating NSs), it can still migrate to a supramassive star by mass loss through early winds while still supported by differential rotation (see below). Supramassive NSs are typically long-lived and the exact lifetime depends on the timescale to remove a significant fraction of the rotational energy (e.g., via dipole spin down). Hence, it is conceivable that in the vast majority of BNS mergers a long-lived NS is formed, which supports the magnetar model.

From simulations it is known, however, that at early times the BNS merger remnant is characterized by a high degree of differential rotation and thus substantial magnetic field rearrangement and amplification. These conditions are very different from pure dipole spin down of a uniformly rotating magnetar, as assumed in most magnetar models. This leads us to investigate this early phase of a differentially rotating BNS merger remnant by fully general-relativistic magnetohydrodynamic simulations. We elaborate here on recent results of such simulations [18], which revealed the generation of a very dense, highly magnetized wind that carries sufficient Poynting flux to explain typical luminosities of early X-ray afterglows. The present paper focuses on discussing the wind properties in more detail and its implications for SGRBs and their X-ray afterglows. 

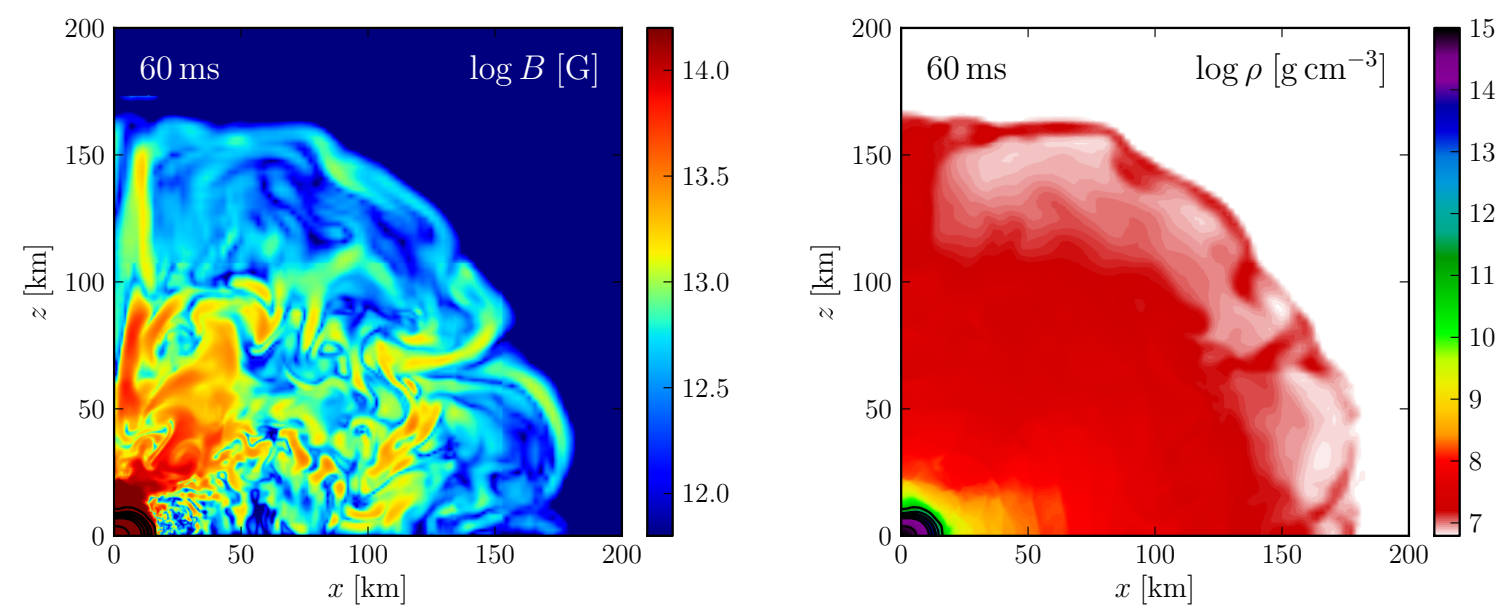

Figure 1: Magnetic field strength (left) and rest-mass density (right) of the wind from a differentially rotating NS (corresponding to model rand of [18]; the star is indicated by black density contours in the lower-left corners). These snapshots showing a very dense, highly isotropic and highly magnetized wind are taken at the end of the simulation.

\section{Initial data and numerical setup}

Employing the general-relativistic ideal magnetohydrodynamics code WHISKYMHD [19, 20, 21] combined with the publicly available Einstein Toolkit and its spacetime evolution code MCLACHLAN [22], models of hypermassive neutron stars constructed with the RNS code [23] were evolved, which we endowed with different initial magnetic field geometries (for details, see [18]). These models are representative of the typical outcome of a BNS merger, once the merger remnant has settled down to a roughly axisymmetric state and assuming that the merger has not led to a prompt collapse to a BH. Here, we elaborate, in particular, on model rand of [18], which has a "random" initial magnetic field geometry inside and outside the star and which we consider to most closely resemble the actual magnetic field geometry resulting from a BNS merger. It is important to stress that our conclusions in this paper and that of [18] mostly depend on differential rotation and thus also apply to differentially rotating supramassive NSs (which together with hypermassive NS are the most likely result of BNS mergers, see Section 1).

\section{Evolution, mass ejection, electromagnetic emission}

The early evolution of a differentially rotating BNS merger remnant is characterized by magnetic winding: poloidal magnetic fields in the interior of the star are "wound up", producing a strong toroidal component and enhancing the magnetic pressure. Rotational energy is thus converted into magnetic energy. We find that within a few average rotational periods, the increase of magnetic pressure in the surface layers of the star is sufficient to overcome the gravitational binding of material in the vicinity of the stellar surface. This results in a highly magnetized, highly isotropic and very dense wind of matter from the NS that heavily pollutes the environment with baryons (cf. 

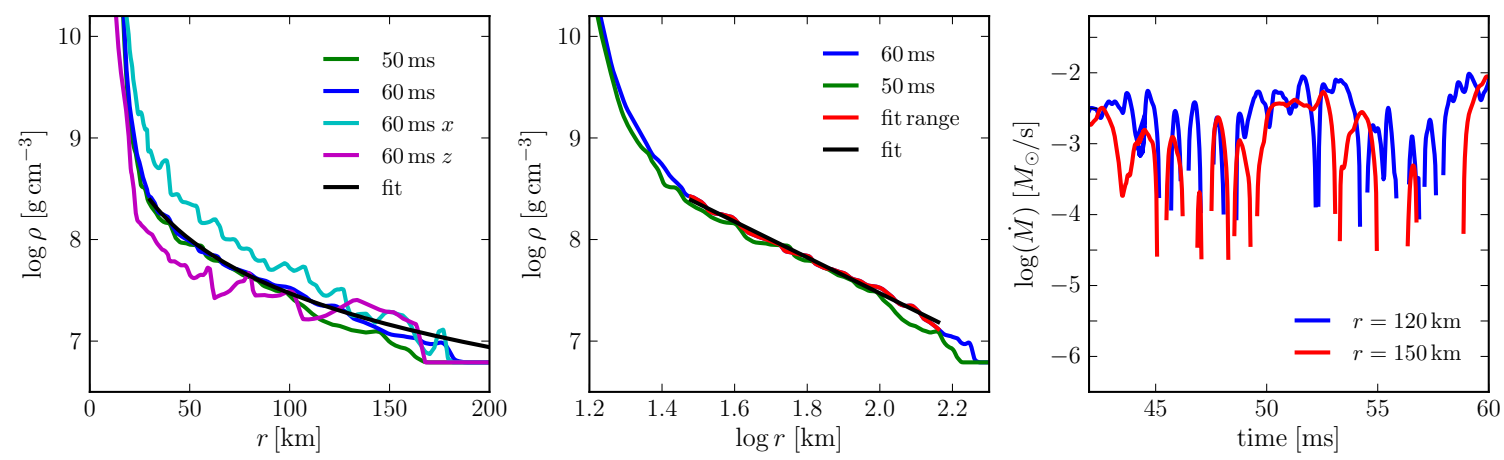

Figure 2: Left and middle panel: rest-mass density profiles of the wind from a differentially rotating NS (corresponding to model rand of [18]) at different times towards the end of the evolution (density profiles along the $x$ and $z$ axes in cyan and purple, respectively, polar angle averaged profiles in green and blue). The black line shows a fit $\rho \propto r^{-2}$ to the angular averaged profiles. Right panel: instantaneous mass-loss rate associated to the wind as measured at different radii during the evolution.

Figure 1). ${ }^{1}$ This environment of the NS is very different from an essentially baryon-fee pulsar wind that is usually hypothesized in magnetar models.

A global measure of the baryon pollution is depicted in the rightmost panel of Figure 2, which shows the total mass crossing a coordinate sphere of radius $r=120 \mathrm{~km}$ and $r=150 \mathrm{~km}$ from the centre of the star per unit time during the evolution of the simulation. The wind at these distances is still rather turbulent during the later part of the evolution, which results in a noisy mass-loss rate. Nevertheless, the mass-loss rates as measured at different distances from the star roughly agree and yield a stationary mass-loss rate of a few in $10^{-3}$ solar masses per second $\left(\dot{M} \sim 10^{-3} \mathrm{M}_{\odot} \mathrm{s}^{-1}\right)$.

The morphology of the wind is depicted in the left and middle panel of Figure 2 in terms of the rest-mass density profiles. The directionally averaged profiles ${ }^{2}$ at different times (e.g., at $50 \mathrm{~ms}$ and $60 \mathrm{~ms}$ ) agree very well up to the outer radius of the bulk of the ejected matter at that particular time. A fit to these profiles reveals a characteristic average wind density profile $\rho \propto r^{-2}$ that is constant in time. According to mass conservation, we have at fixed radius

$$
\dot{M} \equiv\left|\frac{\partial M(r, t)}{\partial t}\right|=4 \pi r^{2} \rho(r, t) v(r, t),
$$

where $M$ is the amount of mass inside the volume of radius $r$ and $v(r, t)$ is the radial velocity of the wind at time $t$ and radius $r$. Combining $\rho(r, t)=\rho(r) \propto r^{-2}$ and $\dot{M}(r, t)=\dot{M}=$ const thus yields $v(r, t)=v_{0}=$ const, i.e. an overall bulk speed of the wind that is constant in time and space. Assuming $\dot{M} \simeq 5 \times 10^{-3} \mathrm{M}_{\odot}$ (see above and Figure 2), we obtain a subrelativistic wind expansion speed for the model considered, $v_{0} \simeq 0.03 c$, where $c$ is the speed of light.

Although the mass-loss rate is roughly constant during the timescale of up to $60 \mathrm{~ms}$ that we can access through our simulations, it is expected to change significantly at later times. The timescale for a change of $\dot{M}$ and thus for a change of the wind properties depends on the timescale for removal

\footnotetext{
${ }^{1}$ In the case of a dominant dipole moment in the initial magnetic field structure, we expect an additional collimated wind component directed along the rotation axis [18].

${ }^{2}$ The system is very close to axisymmetry and averaging over the polar angle is sufficient to obtain a directionally averaged profile.
} 
of differential rotation (see Section 4). Furthermore, neutrinos radiated from the hot interior of the NS can heat and drive an outflow from the surface of the NS and thus lead to additional mass loss (e.g., $[24,25]$ ). Depending on the magnetic field strength and the rotation period, however, the magnetically-driven wind discussed here may well be the dominant process for mass loss.

The Poynting flux associated to the magnetically-driven wind in our simulations corresponds to stationary electromagnetic (EM) luminosities

$$
L_{\mathrm{EM}} \simeq 10^{48} \bar{B}_{15}^{2} R_{\mathrm{e}, 6}^{3} P_{-4}^{-1} \mathrm{erg} \mathrm{s}^{-1},
$$

where $\bar{B}$ is the average magnetic field strength in the outer layers of the star once the system has reached its stationary state, $R_{\mathrm{e}}$ denotes the equatorial radius, and $P$ is the (central) spin period [18]. This relation is universal in the sense that it does not depend on the initial magnetic field geometry and it is different from dipole spin-down emission considered in the magnetar model, in which case $L_{\mathrm{EM}} \propto B_{\mathrm{p}}^{2} R_{\mathrm{e}}^{6} P^{-4}$, where $B_{\mathrm{p}}$ is the dipolar field strength at the pole. Assuming that only a small fraction $\eta \equiv L_{\mathrm{EM}}^{\mathrm{obs}} / L_{\mathrm{EM}} \sim 0.01-0.1$ of the luminosity (Equation 3.2) is converted into X-rays, the observed X-ray luminosities $L_{\mathrm{EM}}^{\mathrm{obs}} \sim 10^{46}-10^{51} \mathrm{erg} \mathrm{s}^{-1}$ of extended emission and X-ray plateaus of SGRBs (e.g., [7, 8]) are obtained by magnetic field strengths $\bar{B} \sim 10^{14}-10^{17} \mathrm{G}$, which are likely produced during and after a BNS merger $[18,26]$.

\section{Conclusions: Consequences for SGRBs and X-ray afterglows}

The timescale over which the baryon-loaded wind and its associated EM luminosity can be sustained depends on the timescale for removal of differential rotation and is uncertain. An order of magnitude estimate is provided by the Alfvén timescale [27], which corresponds to $\sim 1-10$ s for the magnetic fields considered here and which is in agreement with an estimate $\Omega / \dot{\Omega} \lesssim 10$ s for the timescale of change of the angular velocity profile in our simulations. Differential rotation is completely removed within a few to several of these timescales. The overall duration of the process as well as the luminosities mentioned above are compatible with the observed extended emission of SGRBs (lasting $\lesssim 100$ s) and thus make the magnetically-driven wind a promising alternative model to explain such emission.

The prompt SGRB emission is thought to be collimated and thus beamed away from us in the vast majority of events. Furthermore, the observed SGRB rate within the sensitivity volume of advanced LIGO and Virgo is very small $\left(\lesssim 1 \mathrm{yr}^{-1}\right.$ [28]). Therefore, identifying potential isotropic EM emission from BNS mergers is of utmost importance. The isotropic EM emission found here potentially represents such an important counterpart to the GW signal of BNS mergers.

Although the initial phase of differential rotation of the BNS merger remnant is rather short, the total amount of mass lost via the magnetically-driven wind can be substantial. The ejected matter creates an optically thick environment that will likely affect the emission properties of the system in the following evolution, once the star has settled down to uniform rotation. At this stage, the NS might lose rotational energy via spin-down radiation, and the resulting X-ray afterglow radiation would be the product of a reprocessing of this radiation by the confining envelope of ejecta matter (cf. [29, 30]). The associated spectrum could carry such a signature, e.g., thermalization of (part of) the non-thermal spin-down emission by the optically thick ejecta material. 
As the presence of a magnetically-driven wind only depends on differential rotation, which is generic to BNS merger remnants, the associated baryon pollution in the polar regions is also generally expected. This severely hampers the production of a relativistic jet through accretion of a remnant torus around the newly formed NS. Hence, if the presence of long-lasting X-ray afterglows requires a long-lived NS remnant, an alternative explanation for the generation of the SGRB itself is likely required. Such a scenario that explains the production of long-lasting X-ray afterglows and the prompt SGRB emission in a common phenomenology has recently been proposed [29, 30] (for an alternative proposal, see [31]). However, full simulations of BNS mergers will be needed in the future to better characterize the magnetically-driven wind. These simulations will also help to shed light on whether or not a relativistic jet can be launched at the merger of a BNS system forming a long-lived NS.

R.C. is supported by MIUR FIR grant No. RBFR13QJYF.

\section{References}

[1] G. M. Harry, LIGO Scientific Collaboration, Advanced LIGO: the next generation of gravitational wave detectors, CQG 27 (2010) 084006.

[2] T. Accadia, et al., Status of the Virgo project, CQG 28 (2011) 114002.

[3] D. Eichler, et al., Nucleosynthesis, neutrino bursts and gamma-rays from coalescing neutron stars, Nature 340 (1989) 126.

[4] R. Narayan, B. Paczynski, \& T. Piran, Gamma-ray bursts as the death throes of massive binary stars, ApJL 395 (1992) L83.

[5] L. Rezzolla, et al., The missing link: merging neutron stars naturally produce jet-like structures and can power short gamma-ray bursts, ApJL 732 (2011) L6.

[6] V. Paschalidis, M. Ruiz, \& S. L. Shapiro, Relativistic simulations of black hole-neutron star coalescence: the jet emerges, (2014) [astro-ph/1410.7392].

[7] A. Rowlinson, et al., Signatures of magnetar central engines in short GRB light curves, MNRAS $\mathbf{4 3 0}$ (2013) 1061.

[8] B. P. Gompertz, P. T. O'Brien, \& G. A. Wynn, Magnetar powered GRBs: explaining the extended emission and X-ray plateau of short GRB light curves, MNRAS 438 (2014) 240.

[9] N. Gehrels, et al., The Swift gamma-ray burst mission, ApJ 611 (2004) 1005.

[10] B. D. Metzger, E. Quataert, \& T. A. Thompson, Short-duration gamma-ray bursts with extended emission from protomagnetar spin-down, MNRAS 385 (2008) 1455.

[11] N. Bucciantini, et al., Short gamma-ray bursts with extended emission from magnetar birth: jet formation and collimation, MNRAS 419 (2012) 1537.

[12] B. Zhang, \& P. Mészáros, Gamma-ray burst afterglow with continuous energy injection: signature of a highly magnetized millisecond pulsar, ApJL 552 (2001) L35.

[13] H.-J. Lü, et al., The millisecond magnetar central engine in short GRBs, (2015) [astro-ph/1501.02589].

[14] P. B. Demorest, et al., A two-solar-mass neutron star measured using Shapiro delay, Nature $\mathbf{4 6 7}$ (2010) 1081. 
[15] J. Antoniadis, et al., A massive pulsar in a compact relativistic binary, Science 340 (2013) 448.

[16] K. Belczynski, et al., The lowest-mass stellar black holes: catastrophic death of neutron stars in gamma-ray bursts, ApJL $\mathbf{6 8 0}$ (2008) L129.

[17] J.-P. Lasota, P. Haensel, \& M. A. Abramowicz, Fast rotation of neutron stars, ApJ, 456 (1996) 300.

[18] D. M. Siegel, R. Ciolfi, \& L. Rezzolla, Magnetically driven winds from differentially rotating neutron stars and X-ray afterglows of short gamma-ray bursts, ApJL 785 (2014) L6.

[19] B. Giacomazzo, \& L. Rezzolla, WhiskyMHD: a new numerical code for general relativistic magnetohydrodynamics, CQG 24 (2007) S235.

[20] B. Giacomazzo, L. Rezzolla, \& L. Baiotti, Accurate evolutions of inspiralling and magnetized neutron stars: equal-mass binaries PRD 83 (2011) 044014.

[21] B. Giacomazzo, \& R. Perna, Formation of stable magnetars from binary neutron star mergers, ApJL 771 (2013) L26.

[22] F. Löffler, et al., The Einstein Toolkit: a community computational infrastructure for relativistic astrophysics, CQG 29 (2012) 115001.

[23] N. Stergioulas, \& J. L. Friedman, Comparing models of rapidly rotating relativistic stars constructed by two numerical methods, ApJ 444 (1995) 306.

[24] B. D. Metzger, T. A. Thompson, \& E. Quataert, On the Conditions for neutron-rich gamma-ray burst outflows, ApJ 676 (2008) 1130.

[25] L. Dessart, et al., Neutrino signatures and the neutrino-driven wind in binary neutron star mergers, ApJ 690 (2009) 1681.

[26] D. M. Siegel, et al., Magnetorotational instability in relativistic hypermassive neutron stars, PRD $\mathbf{8 7}$ (2013) 121302(R).

[27] S. L. Shapiro, Differential rotation in neutron stars: magnetic braking and viscous damping, ApJ 544 (2000) 397.

[28] B. D. Metzger, \& E. Berger, What is the most promising electromagnetic counterpart of a neutron star binary merger?, ApJ 746 (2012) 48.

[29] R. Ciolfi, \& D. M. Siegel, Short gamma-ray bursts in the "time-reversal” scenario, ApJL 798 (2015) L36.

[30] R. Ciolfi, \& D. M. Siegel, Short gamma-ray bursts from binary neutron star mergers: the time-reversal scenario, in proceedings of Swift: 10 Years of Discovery, POS (SWIFT 10 ) 108 (2015) [astro-ph/1505.01420].

[31] L. Rezzolla, \& P. Kumar, A novel paradigm for short gamma-ray bursts with extended X-ray emission, ApJ 802 (2015) 95. 